2. - Über den Variabilitätsbereich der Fourier'schen Konstanten von positiven harmonischen Funktionen, Rend. Circ. Mat. Palermo 32 (1911), 193-217.

3. G. Herglotz, Über Potenzreihen mit positivem reellen Teil im Einheitskreis, Ber. Sachs. Akad. Wiss. Leipzig Math.-Phys. K1. 63 (1911), 501-511.

4. C. Loewner, Üntersuchungen über die Verzerrung bei Konformen Abbildungen des Einheitskreises $|z|<1$ die durch Funktionen mit nicht verschwindender Ableitung geliefert werden, Ber. Sachs. Akad. Wiss. Leipzig, Math.-Phys. Kl. 69 (1917), 89-106.

5. Z. Nehari, Conformal mapping, McGraw-Hill, New York, 1952.

6. R. Nevanlinna, Eindeutige Analytische Funktionen, J. W. Edwards, Ann Arbor, Michigan, 1944.

UNIVERSITY OF KENTUCKY

\title{
VANISHING CENTRAL DIFFERENCES
}

RICHARD F. DEMAR

Given a sequence $\left\{a_{n}\right\}_{n=0}^{\infty}$ of complex numbers, a number of theorems have been proved concerning implications of the vanishing of certain differences $\Delta^{n} a_{0}$ if the given sequence satisfies some growth restriction. The first such result, proved by Agnew [1], states that if $\left\{a_{n}\right\}$ is bounded and $\Delta^{2 n} a_{0}=0$ for all $n$, then $a_{n}=0$ for all $n$. If all the odd differences are zero, the sequence is constant. Fuchs [6] proved the following: Let $a_{n}=o\left(n^{k}\right)$ for some positive number $k$, and let $n_{j}$ be a subsequence of the positive integers such that if $n(R)$ is the number of $n_{j}<R$, then $n(R) \geqq R / 2$ for $R>R_{0}$. If $\Delta_{j}^{n} a_{0}=0$ for all $n_{j}$, then $a_{n}=p(n)$ where $p(x)$ is a polynomial of degree less than $k$. Buck [4] assumed only that lim sup $\left|a_{n}\right|^{1 / n}<1$ and $\Delta^{n} a_{0}=0$ for all $n$ belonging to a set of positive integers of density $d>\frac{1}{3}$ and proved there is a function $f$ of exponential type whose growth function $h(\theta)$ satisfies $h( \pm \pi / 2)<\pi$ such that $f(n)=a_{n}$ for all $n$. In this paper, we show that if the given sequence is extended to $\left\{a_{n}\right\}_{n=-\infty}^{\infty}$ by letting $a_{-n}=a_{n}$, then the vanishing of certain of the even central differences $\Delta^{2 n} a_{-n}$ has similar implications. Or, letting $a_{-n}=-a_{n-1}$, vanishing of odd differences $\Delta^{2 n-1} b_{-n}$ gives similar results.

If $G$ is a connected set, let $K[G]$ denote the class of all entire functions of exponential type whose conjugate indicator diagrams $D(f)$ are contained in $G$. If $G$ is the rectangle $\{x+i y|| x|\leqq a ;| y \mid \leqq c\}$, then $K[a, c]$ will be used for $K[G]$. Let $C_{z, n}$ denote the polynomial $z(z-1) \cdots(z-n+1) / n !$

Certain results concerning the sequence $\left\{\mathscr{L}_{n}\right\}$ of Stirling functionals given by $\mathscr{L}_{n}(f)=\Delta^{n} f(-n / 2)$ will be needed. These functionals

Presented to the Society, January 24, 1961, under the title, A theorem on vanishing differences; received by the editors November 24,1961 and, in revised form, December $12,1961$. 
have the representation

$$
\Delta^{n} f(-n / 2)=\frac{1}{2 \pi i} \int_{\Gamma}\left(e^{\zeta / 2}-e^{\zeta / 2}\right)^{n} F(\zeta) d \zeta
$$

where $F$ is the Borel (Laplace) transform of $f$ and $\Gamma$ is any simple contour enclosing the conjugate indicator diagram $D(f)$. Let $B$ be the set of all $\zeta$ satisfying $\left|e^{\zeta / 2}-e^{\zeta / 2}\right|<2$. Then $B$ is a convex, lensshaped region, symmetric about the origin whose boundary has vertices at $\pm \pi i$ and crosses the real axis at $\pm \log (3+2 \sqrt{ } 2)$. For $f$ in $K[B]$, Buck [3] showed that

$$
f(z)=\sum_{n=0}^{\infty} \Delta^{n} f(-n / 2)(z / n) C_{z+n / 2-1, n-1},
$$

convergent for all $z$. The author [5] showed that for a given sequence $\left\{c_{n}\right\}$ of complex numbers, there is a function $f$ in $K[B]$ such that $\Delta^{n} f(-n / 2)=c_{n} ; n=0,1,2, \cdots$ if and only if $\lim \sup \left|c_{n}\right|^{1 / n}<2$. If we let $G(t)=\sum c_{n} t^{n}$, then $f$ has the representation

$$
f(z)=\frac{1}{2 \pi i} \int_{E} \frac{G(t)}{t} \exp \left[2 z \sinh ^{-1} 1 /(2 t)\right] d t
$$

where $E$ is a simple contour contained in the region of regularity of $G$ and enclosing the disk $|t| \leqq \frac{1}{2}$. Then the conjugate indicator diagram $D(f)$ is contained in the convex hull of the image of $E$ under the $\operatorname{map} \zeta=2 \sinh ^{-1} 1 /(2 t)$.

THEOREM 1. Let $\left\{b_{n}\right\}_{n--\infty}^{\infty}$ be an even sequence of complex numbers such that $\lim \sup \left|b_{n}\right|^{1 / n} \leqq 1$. Suppose there is a set $A$ of positive integers of density $d>0$ such that for all $n$ in $A, \Delta^{2 n} b_{-n}=0$. Then $\sum \Delta^{2 n} b_{-n}(z / 2 n) C_{z+n-1, n-1}$ converges to an even function $f$ in $K[B]$ and $f(n)=b_{n} ; n=0, \pm 1, \pm 2, \cdots$.

We need the following lemma.

Lemma. For a sequence $\left\{b_{n}\right\}_{n--\infty}^{\infty}$, define $Q(t)$ formally by

$$
Q(t)=\sum_{n=0}^{\infty} \sum_{k=0}^{n} C_{n, k} b_{-n+2 k} t^{n}
$$

and define $P(z)$ by $P(z)=(1+2 z)^{-1} Q(z /(1+2 z))$. Then formally $P(z)$ $=\sum \Delta^{2 n} b_{-n} z^{n}$.

Proof. ${ }^{1}$ Let $E$ be an operator defined for a sequence $a=\left\{a_{k}\right\}$ by $E(a)(k)=a(k+1)$. Then $\Delta=E-1$, and we have

\footnotetext{
1 This proof was suggested by Professor R. C. Buck.
} 
RICHARD F. DEMAR [February

$$
\begin{aligned}
Q(t) & =\sum_{n=0}^{\infty} \sum_{k=0}^{n} C_{n, k} b_{-n+2 k} t^{n} \\
& =\sum_{n=0}^{\infty} t^{n} E^{-n} \sum_{k=0}^{n} C_{n, k} E^{2 k} b_{0} \\
& =\sum_{n=0}^{\infty} t^{n}\left(E+E^{-1}\right)^{n} b_{0} \\
& =\frac{1}{1-t\left(E+E^{-1}\right)} b_{0} . \\
Q(z /(1+2 z)) & =(1+2 z) \frac{1}{1-z\left(E-2+E^{-1}\right)} b_{0} \\
& =(1+2 z) \sum_{n=0}^{\infty} z^{n}\left(E-2+E^{-1}\right)^{n} b_{0} \\
& =(1+2 z) \sum_{n=0}^{\infty} \Delta^{2 n} b_{-n} z^{n} .
\end{aligned}
$$

Proof of Theorem 1. Since

$$
\lim \sup \left|b_{n}\right|^{1 / n} \leqq 1, \quad \lim \sup \left|\sum_{k=0}^{n} C_{n, k} b_{-n+2 k}\right| \leqq 2 .
$$

Thus $Q(t)$ is regular in the disk $|t|<\frac{1}{2}$. Then from its definition $P(z)$ is regular for $|z /(1+2 z)|<\frac{1}{2}$ or $|z|<\left|z+\frac{1}{2}\right|$ which is the set of all $z$ whose real part is greater than $-\frac{1}{4}$. Let $G(z)=P\left(z^{2}\right)=\sum \Delta^{2 n} b_{-n} z^{2 n}$. Then $G$ is regular for all $z$ such that $R\left(z^{2}\right)>-\frac{1}{4}$; i.e., for $z$ in the region containing the origin and bounded by the equilateral hyperbola $y^{2}-x^{2}=\frac{1}{4}$ where $z=x+i y$. Let $r_{0}$ be the radius of convergence of $\sum \Delta^{2 n} b_{-n} z^{2 n}$. Then $r_{0} \geqq \frac{1}{2}$. Since $G$ is even, $G(z)=\sum c_{n} z^{n}$ where $c_{2 n+1}=0$ and $c_{2 n}=\Delta^{2 n} b_{-n}$. Then from the hypothesis, $c_{n}=0$ for all $n$ belonging to a set of density $d^{\prime}>\frac{1}{2}$. Thus, by Polya's density theorem [7], $G$ has a singularity on every arc of $|z|=r_{0}$ of opening $2 \pi(1-d)$ and this is less than $\pi$. But if $r_{0}=\frac{1}{2}$, the only possible singularities are at $i / 2$ or $-i / 2$; so $r_{0}>\frac{1}{2}$. Then, by the results on Stirling functionals quoted earlier, there is a function $f$ in $K[B]$ such that $\Delta^{2 n} f(-n)$ $=\Delta^{2 n} b_{-n}$ for all $n$, and $f(z)=\sum \Delta^{2 n} b_{-n}(z / 2 n) C_{z+n-1, n-1}$ convergent for all $z$. Since $(z / 2 n) C_{z+n-1, n-1}$ is even for each $n, f$ is even. It can be shown by induction that $f(n)=b_{n} ; n=0, \pm 1, \pm 2, \cdots$, using the fact that $\Delta^{2 n} f(-n)=\Delta^{2 n} b_{-n}$ for each $n$. Q.E.D.

TheOREM 2. In Theorem 1 , if $d \geqq \frac{1}{2}$, then $f$ is of zero type.

Proof. If $d \geqq \frac{1}{2}$, then $G(z)=\sum \Delta^{2 n} b_{-n} z^{2 n}$ has zero coefficients for 
all $n$ belonging to a set of positive integers of density at least $\frac{3}{4}$. Then, by Pólya's density theorem, $G$ has a singularity on every arc of its circle of convergence of opening $\pi / 2$. But $G$ is regular for all $z=r e^{i \theta}$ with $\theta \leqq \pi / 4$, so $G$ is entire. Then in representation (1) of $f$, the contour $E$ can be taken as a circle of arbitrarily large radius, so that its image under the map $\zeta=2 \sinh ^{-1} 1 /(2 t)$ can be made to lie in an arbitrarily small disk about the origin. Therefore $D(f)$ is the origin, i.e., $f$ is of zero type. Q.E.D.

CoROllaRy 3. In Theorem 1 , if $d \geqq \frac{1}{2}$ and $b_{n}=o\left(n^{k}\right)$ as $n \rightarrow \infty$ for some $k>0$, then $f$ is a polynomial of degree less than $k$.

Proof. The function $f$ is of zero type and since $f$ is even, $f(n)$ $=o\left(|n|^{k}\right)$ as $n \rightarrow \pm \infty$; so $f$ is a polynomial of degree less than $k$ [2, p. 183].

CoROllary 4. In Corollary 3 , if $\left\{b_{n}\right\}$ is bounded then it is a constant sequence.

Thus we have obtained theorems analogous to those of Buck, Fuchs, and Agnew referred to at the beginning.

Since, for an odd sequence $\left\{c_{n}\right\}_{n=-\infty}^{\infty}, \Delta^{2 n} c_{-n}=0$ for all $n$, we have the following:

CoROllaRy 5. If any bounded sequence $\left\{b_{n}\right\}_{n=-\infty}^{\infty}$ has $b_{0}=0$ and $\Delta^{2 n} b_{-n}=0$ for all $n$ belonging to $a$ set of positive integers of density $d \geqq \frac{1}{2}$, then $\left\{b_{n}\right\}$ is an odd sequence.

For a sequence $\left\{b_{n}\right\}_{n=-\infty}^{\infty}$ such that $b_{-n}=-b_{n-1} ; n=1,2,3, \cdots$, we obtain theorems identical with those above except that the even differences are replaced by odd differences $\Delta^{2 n-1} b_{-n}$ and the interpolating function is an odd function. The proofs of these theorems are almost the same as the proofs of the above theorems.

\section{BIBLIOGRAPHY}

1. R. P. Agnew, On sequences with vanishing even or odd differences, Amer. J. Math. 66 (1944), 339-340.

2. R. P. Boas, Entire functions, Academic Press, New York, 1954.

3. R. C. Buck, Interpolation series, Trans. Amer. Math. Soc. 64 (1948), 283-298.

4. - On admissibility of sequences and a theorem of Polya, Comment. Math. Helv. 27 (1953), 75-80.

5. R. F. DeMar, Existence of interpolating functions of exponential type, Trans. Amer. Math. Soc. 105 (1962), 359-371.

6. W. H. J. Fuchs, $A$ theorem of finite differences with application to the theory of Hausdorff summability, Proc. Cambridge Philos. Soc. 40 (1944), 188-198.

7. G. Pólya, Über Lucken und Singularitäten von Potenzreihen, Math. Z. 29 (1929), 549-640.

Miami University 